\title{
The multifractal of ultra low carbon steels containing titanium organizations
}

\author{
Shengli Cao ${ }^{1, \mathrm{a}}$, Qingjun Zhang ${ }^{1,2, \mathrm{~b} *}$, Liguang Zhu ${ }^{1, \mathrm{c}}$, Wenling $\mathrm{Mo}^{3, \mathrm{~d}}$, \\ Chunliang Yan ${ }^{2, e}$, Xiaoyan $\mathrm{Wu}^{1, \mathrm{f}}$
}

1. Hebei Province High Quality Steel Continuous Casting Engineering Technology Research Center, College of Metallurgy \& Energy, North China University of Science and Technology, Tangshan 063009, China;

\section{Analysis and Testing Center, North China University of Science and Technology, Tangshan 063009, China;}

3. Yisheng College, North China University of Science and Technology, Tangshan 063009, China ahblhcsl@126.com, ${ }^{\mathrm{b}}$ zhangqingjun@heuu.edu.cn, ${ }^{\mathrm{c}}$ zhulg@heuu.edu.cn, ${ }^{\mathrm{d}} \mathrm{mwl} @$ heuu.edu.cn, e360558944@qq.com, '947033946@qq.com

Keywords: multifractal; pulsed magnetic field; microstructure

Abstract. Steel samples under different pulse magnetic field are produced using self-developed pulsed magnetic field generator, and the discharge voltage of the pulsed magnetic field selected from the experiment is $0 \mathrm{~V}, 40 \mathrm{~V}, 80 \mathrm{~V}$, and $100 \mathrm{~V}$ four gradient levels. The morphology of the microstructure is photographed by metallographic microscope, then the calculation of the multi fractal software, which is designed by Visual $\mathrm{C}++$, of the optical image input is carried out. The distribution patterns of different pulse magnetic fields have been quantitatively characterized by the results of the calculation. With the increase of the discharge voltage, (Multifractal spectrum width) decreases from 0.481338 to 0.439.5 and then increases to 0.545596 , which explains process of the change from uniform to uniform to uneven and more complicated organizational structure; (multifractal spectrum) $>0$, the fine microstructure dominated, the increased proportion of intracrystalline ferrite.

\section{Introduction}

Recent studies show that pulsed magnetic field can control solidification process and effectively refine the metal solidification microstructure ${ }^{[1-3]}$. The oxide metallurgy technology can use inclusions in steel pinning movement of grain boundaries at high temperatures to inhibit grain growth and promote the nucleation of intragranular ferrite to refine the microstructure of the steel ${ }^{[4]}$. The steel in this paper is to use the dual thinning effect of pulsed magnetic field and oxide metallurgical technology. It is hard to describe with the traditional quantitative method because of the irregularity of the structure of the steel structure. The simple fractal dimension is only the overall characterization of the surface morphology and not detailed description of details ${ }^{[5]}$. Multifractal can reflect more details of material surface and more real description of the surface morphology of the material ${ }^{[6]}$. The multifractal spectrum of organizations at different discharge voltage are quantitatively calculated and the influence of pulsed magnetic field on microstructure of steel is studied in more detail using self-designed multi-fractal software.

\section{Experiment}

In experiment, steel samples under pulse magnetic field with different discharge voltage are produced using self-developed pulsed magnetic field generator. In order to study the effect of pulsed magnetic field under different discharge voltage by using multifractal method, the discharge voltage of the experiment consists of $0 \mathrm{~V}, 40 \mathrm{~V}, 80 \mathrm{~V}$ and $100 \mathrm{~V}$ four gradient level. One $10 \mathrm{~mm} \times 10 \mathrm{~mm}$ cross section for the samples is obtained by wire cutting. The samples are polished, ultrasonic cleaning, 
drying and Nitrate corrosion and the morphology of the microstructure is photographed by metallographic microscope, and then the calculation of the multi fractal software of the optical image input is carried out.

\section{Results and discussion}

In order to more systematically study the effect of pulsed magnetic field under different discharge voltage, the surface morphology of the $0 \mathrm{~V}, 40 \mathrm{~V}, 80 \mathrm{~V}$ and $100 \mathrm{~V}$ is photographed, As shown in Figure 1. With increasing discharge voltage, the surface morphology of the microstructure gradually becomes micro organization from relatively coarse microstructure and the metallographic structure becomes more complex, which is mainly due to the increase of the discharge voltage and the generation of large amount of acicular ferrite structure.

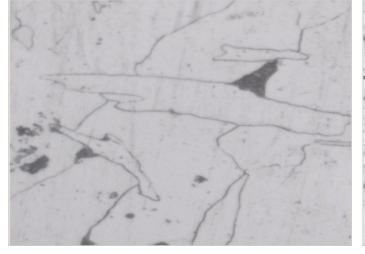

(a)

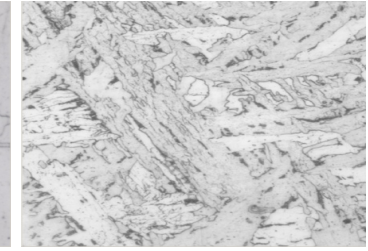

(b)

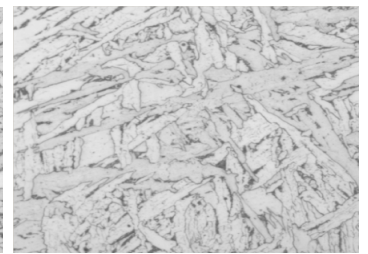

(c)

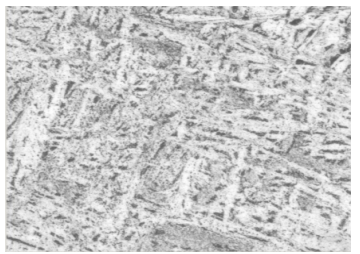

(d)

Fig. 1 Microstructure of different discharge voltages(a:0v;b:40v;c:80v;d:100v)

In this paper, the box countin method is used to calculate and count. First, microstructure pictures are binarizingly processing by selecting the appropriate threshold. Figure 2 is binarized images of Fig. 1 in the conversion threshold with respectively 116, 123, 105, 124.

Fig. 2 is respectively covered with the square with a scale of $\varepsilon=\frac{1}{512}, \frac{1}{256}, \frac{1}{128}, \frac{1}{64}, \frac{1}{32}, \frac{1}{16}, \frac{1}{8}$, $\frac{1}{4}, \frac{1}{2}$ and calculate the corresponding probability measure. Define a distribution function $\chi_{\mathrm{q}}(\varepsilon)=\sum$ $\mathrm{P}_{\mathrm{ij}}(\varepsilon)_{\mathrm{q}}=\varepsilon \ln \chi_{\mathrm{q}}(\varepsilon) / \ln \varepsilon$, and If the equation is established, the distribution function $\chi_{\mathrm{q}}(\varepsilon)$ and $\varepsilon$ have a power function relationship and in $\chi_{\mathrm{q}}(\varepsilon) \sim \ln (\varepsilon)$ are a straight line. Namely, it can meet the invariance or self similarity of the scale change and it is suitable for the analysis of multifractal spectrum. $\alpha=\mathrm{d}\left[\ln \chi_{\mathrm{q}}\right.$ $(\varepsilon) / \ln \varepsilon] / \mathrm{d}_{\mathrm{q}}$, which is the singular index, can reflect the singularity of the units in the fractal. Here, $\mathrm{f}(\alpha)$ $=\alpha \mathrm{q}-\ln \chi_{\mathrm{q}}(\varepsilon) / \ln \varepsilon$, which is a multifractal spectrum, reflect fractal dimension of the subset of the same $\alpha$ value. By choosing the appropriate $q$ values and the calculated $\mathrm{P}_{\mathrm{ij}}(\varepsilon)$, we can calculate the $\alpha$ and $\mathrm{f}(\alpha)$, which can quantitatively characterize the complexity of fractal structure ${ }^{[7]}$.

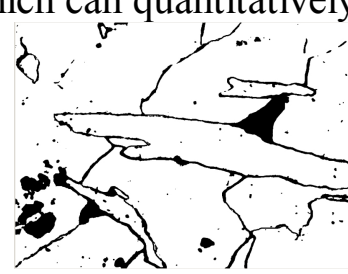

(a)

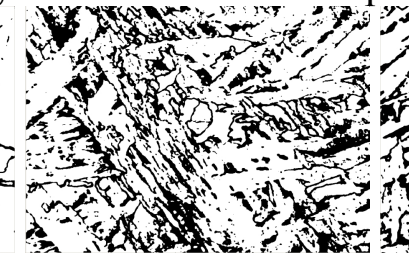

(b)

\begin{abstract}
(c)
\end{abstract}
Fig. 2 Binarized images of Fig. 1(a:0v;b:40v;c:80v;d:100v)
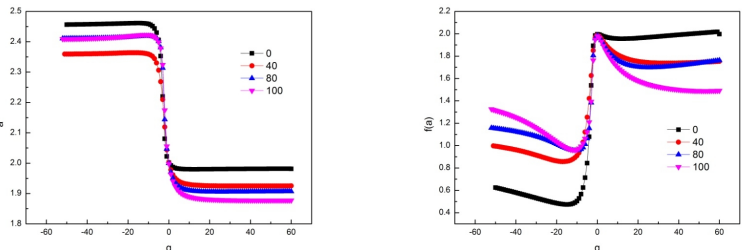

Fig. 3 Curves of $\mathrm{f}(\alpha) \sim \mathrm{q}$ and $\alpha \sim \mathrm{q}$ of Fig. 2

By the relationship curves between $\alpha \sim \mathrm{q}$ and $\mathrm{f}(\alpha) \sim \mathrm{q}$ (Figure 3), we can know, when $|\mathrm{q}|>10$, a almost does not change with the change of q, that is to say, the weight factor q has little effect on the value of multifractal spectrum function, which can be neglected. By the right, when the q ranges from 
-20 to $20, f(\alpha)$ dramatically changes with the change of $q$. Beyond this range of $q, f(\alpha)$ slowly changes with the change of q, which almost can be ignored, consequently, the range of q is from -20 to 20 in this experiment.
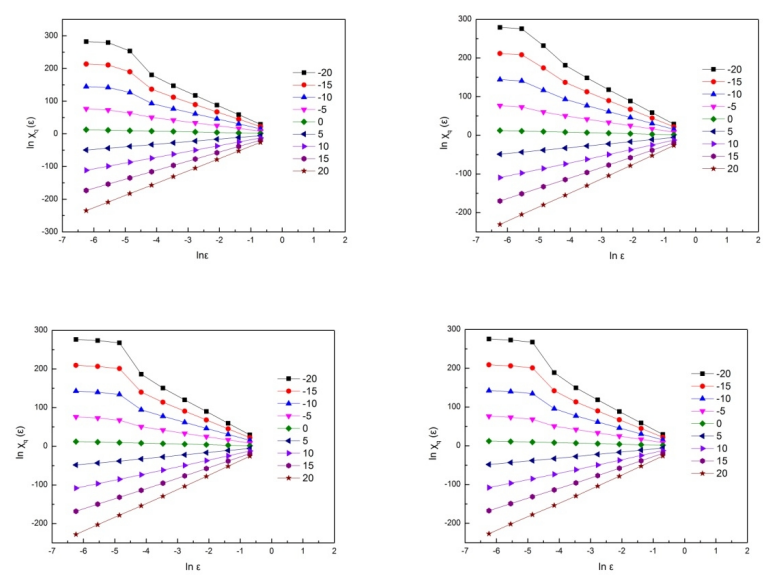

Fig. 4 Curves of $\ln \chi q(\varepsilon) \sim \ln (\varepsilon)$ of Fig. 2

It can be seen from figure 4 that these lines infinitely extended to smaller $\varepsilon$ until gather in the $(0,0)$ point, and in the small change range of $\varepsilon$, the absolute value of the slope is small, that is to say, scaling invariance is not satisfied in all the range of $\varepsilon$. The appearance of this anomaly is related to the fluctuation of the small probability. With the decline of $\varepsilon, \mathrm{P}_{\mathrm{ij}}(\varepsilon)$ may decrease sharply, which causes that $\ln \chi_{\mathrm{q}}(\varepsilon)$ abnormally increases in the range of $\varepsilon$, when $\mathrm{q}$ is negative. An approach to the partial solution of the anomaly is to ignore a part of very small $\mathrm{P}_{\mathrm{ij}}(\varepsilon)$, which is considered to be errors in actual graphics and be neglected. It is explained that a series of samples with different discharge voltage have scale invariance and two dimensional structure of the organization can be studied by multifractal theory.

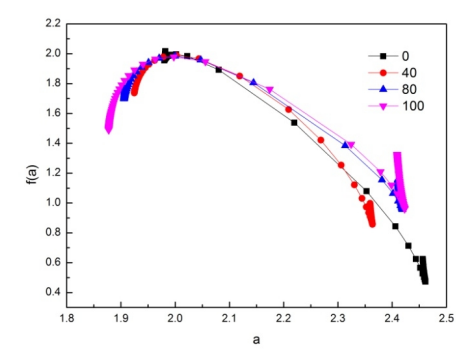

Fig. 5 Curves of multifractal spectrum of Fig. 2

Table 1 Parameters of multifractal spectrum

\begin{tabular}{cccccccc}
\hline $\begin{array}{c}\text { Discharge } \\
\text { voltage }\end{array}$ & $\alpha_{\min }$ & $\alpha_{\max }$ & $\Delta \alpha$ & $\mathrm{f}\left(\alpha_{\min }\right)$ & $\mathrm{f}\left(\alpha_{\max }\right)$ & $\Delta \mathrm{f}(\alpha)$ & $\mathrm{f}(\alpha)_{\max }$ \\
\hline 0 & 1.979911 & 2.461249 & 0.481338 & 1.955625 & 0.474205 & 1.48142 & 2.017399 \\
40 & 1.924788 & 2.363838 & 0.43905 & 1.736664 & 0.85946 & 0.877204 & 1.989283 \\
80 & 1.905614 & 2.418985 & 0.513371 & 1.698587 & 0.956878 & 0.741709 & 1.984146 \\
100 & 1.877441 & 2.423037 & 0.545596 & 1.489196 & 0.960038 & 0.529158 & 1.979204
\end{tabular}

The multifractal spectrum and the main parameters of the structure under the different pulse magnetic field are given in Figure 5 and table 1. As shown above, $\Delta \alpha$ decreases from 0.481338 to 0.439 .5 and then increases to 0.545596 , which shows tissue distribution under different discharge voltage experiences the process from uniform to uniform. This is mainly due to the formation of a large number of acicular ferrite, which makes the structure more complex; $\Delta \mathrm{f}(\alpha)>0$, that is to say, the number of the 
smallest probability subset is greater than the maximum probability subset, which indicates that the fine microstructure is dominated. The $f(\alpha)$ maximum value decreases with the increase of the discharge voltage, which shows the maximum probability is decreasing and the discharge voltage has an obvious effect on the microstructure refinement. This is the same as the observation of the metallographic microscope.

\section{Summary}

(1) The multifractal software can well describe the degree of distribution irregularity and the size distribution of the steel organization.

(2) With the increase of the discharge voltage of the pulsed magnetic field, the microstructure of the Ti bearing carbon steel is from even to more uniform, and then to the uneven. As the appearance of the acicular ferrite, the tissue structure becomes more complex.

(3) The fine microstructure is dominated and the pulse magnetic field has an obvious refinement effect on the microstructure of steel.

\section{Acknowledgments}

The authors are thankful for the support by the Natural Science Foundation of Hebei Province (No. E2013209207), and Scientific Research Project for High-Level Talents in Higher Learning Institution of Hebei Province (No. GCC20142030).

\section{References}

[1] LIAO X L, ZHAI Q J, LUO J, CHEN W J, GONG Y Y.Refining mechanism of the electric current pulse on the solidification structure of pure aluminum[J]. Acta Materialia,2007, 55: 3103-3109.

[2] GONG Y Y, LUO J, JING J X, XIA Z Q, ZHAI Q J. Structure refinement of pure aluminum by pulse magneto-oscillation[J].Materials Science and Engineering A, 2008, 497: 147-152.

[3] LI J, MA J H, GAO Y L, ZHAI Q J. Research on solidification structure refinement of pure aluminum by electric current pulse with parallel electrodes[J]. Materials Science and Engineering A,2008, 490(1/2): 452-456.

[4]LIU Zhong zhu,KUWABARA Mamoru. Up to date progress of technology of oxide metallurgy and its practice[J].Steelmaking,2007,23(3):7-13.

[5] Iasef Md Rian, Jin-Ho Park, Hyung Uk Ahn, Dongkuk Chang. Fractal geometry as the synthesis of Hindu cosmology in Kandariya Mahadev temple[J]. Building and Environment , 2007, 42: 4093.

[6] Olsen L, Winter S. Multifractal analysis of divergence points of deformed measure theoretical Birkhoff averages[J]. Bulletin des Sciences Mathématiques, 2007, 131 (6): 518 .

[7] Feng Dejun. Multifractal analysis of Bernoulli convolutions associated with Salem numbers[J]. Advances in Mathematics, 2012, 229 (5): 3052. 